\title{
Community assembly and the emergence of ecosystem pattern*
}

\author{
SIMON A. LEVIN, JONATHAN DUSHOFF and JUAN E. KEYMER \\ Department of Ecology and Evolutionary Biology, Princeton University, Princeton N.J. 08544-1003, USA.
}

\begin{abstract}
SUMMARY: A fundamental problem challenging natural scientists is to understand how macroscopic patterns, such as population abundance distributions and element ratios, emerge and are sustained in ecosystems, given that evolution typically operates most strongly at the level of individuals and their genomes. How do such patterns persist in the face of evolutionary innovation? In this paper, we explore this issue through dynamical models of community assembly and metapopulation dynamics in dynamic landscapes, and discuss individual-based approaches to the control of element cycles.
\end{abstract}

Key words: community assembly, competitive coexistence, ecosystem pattern, patch dynamics, Redfield ratios, resiliency.

\section{INTRODUCTION}

Ramón Margalef has been an icon for a generation of ecologists. Indeed, ideas he raised nearly half a century ago are still fresh, and only now receiving their due. Building on deep insights into ecological phenomena, especially in the marine environment, he developed and encouraged theoretical approaches that could unify diverse phenomena. Early on, he emphasized the importance of understanding the relationships among processes at different scales of organisation, and the emergence of macroscopic pattern from microscopic phenomena. He understood the relationship between basic and applied work, and was one of the first true biospherists. It is a pleasure and an honour for us to join with the others in this volume in dedicating our work to Ramón.

\footnotetext{
*Received June 27, 2001. Accepted July 17, 2001.
}

\section{COMPLEXITY AND LEVELS OF SELECTION}

Inspired by Ramón Margalef, the focus of this paper is how complexity emerges in ecological communities. It is relatively easy to understand the adaptations of organisms to their surroundings, and in particular how selection operates to buffer their physiologies and life histories against the vagaries of the environment. Ecosystems and the biosphere present more difficult challenges, however, because they are not evolutionary units, selected for robustness (Levin, 1998, 1999). Gaia notwithstanding, the challenge is to understand how patterns emerge and are sustained against evolutionary innovation in these "complex adaptive systems." In such systems, individuals interact locally, and are replicated based on the outcomes of those interactions. No selection principle operates at the level of the whole system, and yet robust patterns emerge that sustain life as we 
know it. There is indeed a coevolutionary process between genome and environment, but it is diffuse coevolution (Ehrlich and Raven, 1964), in which there is no tight linkage between particular organismal traits and particular environmental factors, but in which the collective dynamics of individual evolutionary histories feed back to influence subsequent evolution of the parts.

From this bottom-up perspective, it is natural to ask how biodiversity is maintained, how species abundance patterns emerge, and how nutrient cycles become established and stabilized. We address these questions in turn in the subsequent sections.

\section{SELF-ORGANIZATION IN COMPETITIVE COMMUNITIES}

Understanding the factors that allow competing species to coexist has long been an important question for theoretical biology, and is one of the keys to understanding the tremendous biodiversity of the natural world. Simple models show that the number of species that can be maintained at equilibrium cannot exceed the number of resources (Levins, 1968; Levin, 1970; Tilman, 1990). However, how to count resources is a persistent puzzle, and observed diversities of competitors are often much higher than the number of identifiable resources for which they compete. Possible explanations include partitioning of resources by competitors, tradeoffs involving diseases and predation, or the idea, advanced by Hutchinson (1961), that systems are not at equilibrium. Indeed, Armstrong and McGehee (1980) show how essentially infinitely many species can coexist in environments that fluctuate, or through endogenous oscillations, and Hubbell $(1997,2001)$ shows how coexistence can be maintained in a neutral system, analogous to neutral gene theory, through a balance between speciation (or immigration) and local extinction.

Resource partitioning can take many forms. MacArthur and Levins (MacArthur and Levins, 1967) discussed the partitioning of resources by different species, each with a preferred size range of food. In forests, resources may be partitioned based on differences in micro-sites, which may be determined by biotic or abiotic factors. Species with different life-history strategies may also be able to partition resources in a spatiotemporal manner in an environment with either endogenous or exogenous disturbance (Levin and Paine, 1974). That is, some species may specialize in making use of newly disturbed patches, by growing quickly or by dispersing widely, while other species specialize in more stable patches, eventually outcompeting the early colonizers locally.

\section{Hierarchical competition on landscapes}

A simplistic representation of this phenomenon allows species to compete for a fixed number of sites, and to coexist by virtue of a hierarchical axis trading off competitive ability and reproductive potential. The hierarchical competition model (Levins and Culver, 1971; Horn and MacArthur, 1972; Hastings, 1980) assumes that superior competitors are entirely unaffected by inferior competitors. Thus, if a superior competitor occupies a site, an inferior competitor cannot establish there; conversely, an inferior occupant poses no barrier to a superior competitor establishing on a site (and replacing the occupant).

Following Hastings (1980), Tilman (1994) and Kinzig et al. (1999), we write dynamic equations for the abundance of the species in this system:

$$
\frac{d p_{i}}{d t}=f_{i} p_{i}\left(1-\sum_{j=1}^{i} p_{j}\right)-\left(m_{i}+\sum_{j=1}^{i-1} f_{i} p_{i}\right) p_{i}
$$

Here $f_{\mathrm{i}}$ represents the fecundity and $m_{i}$ mortality of species $i$. For convenience, we give superior competitors lower indices, so that species $i$ is competitively superior to species $j$ whenever $i<j$. The proportion of sites occupied by species $i$ is given by the dynamic variable $p_{i}$. This model (Eq. 1) is the (hierarchical) $n$-species generalization of Levins' (1969) patch-occupancy metapopulation model

$$
\frac{d}{d t} p=f p(1-p)-m p
$$

Clearly, inferior competitors must have some advantage over superior competitors for coexistence to be possible. In metapopulation models, where space is not explicit, this advantage must be either in fecundity $f$, mortality $m$, or some combination of them. Tilman (1994) used higher fecundities for inferior competitors in a model for vegetation dynamics, while Nowak and May (1994) constructed a model for pathogens where inferior competitors instead had lower mortalities. More generally, we can allow both fecundity and mortality to vary along a tradeoff curve, and arrange the species so that inferior competitors (with higher values of $i$ ) also have higher reproductive numbers $R_{i}=f_{i} / m_{i}$ (Dushoff et 
al., submitted). In spatial models, the tradeoff may also involve dispersal distances (Bolker and Pacala, 1999).

The assumption of a strict hierarchy is an extreme one, and can be relaxed (Adler and Mosquera, 2000; Durrett and Levin, 1998), but the hierarchical model's simplicity is appealing both for mathematical analysis (May and Nowak, 1994) and for large-scale simulation (Chave, 2001). Furthermore, there are a variety of systems for which hierarchical competition may at least be a good approximation, ranging from intertidal communities (Levin and Paine, 1974; Paine and Levin, 1981; Quinn, 1982) to perennial grasslands (Tilman, 1994).

Tilman (1994) pointed out that there is no limit to the number of species that can coexist in a hierarchical competition model. No matter how many species are present, a species with sufficiently high fecundity will be able to survive in the open space left by the deaths of the other species. This new species will be the bottom competitor, and so will not drive any other species extinct. However, the number of species that can be added is unlimited only if there are no upper limits to fecundity, which is biologically unrealistic.

\section{Limits to similarity, and species abundace patterns}

Tilman (1994) also showed that there is a "limit to similarity" in this system - that is, species cannot invade a given assemblage if they are too close to superior competitors. In an evolutionary context, however, the limits to diversity also evolve, and packing can grow tighter without limit. May and Nowak (1994) showed that, in a system where new introductions occur sequentially, with the system allowed to come to equilibrium between introductions, the expected number of species surviving increases slowly to infinity (increasing with the log of the number of introductions). They explained this apparent contradiction by showing that, while the density of species at any point on the tradeoff curve approaches an asymptote, as time goes on, the reproductive number $\left(R_{i}=f_{i} / m_{i}\right)$ of the top competitor becomes arbitrarily close to 1 . The small equilibrium population sizes associated with these small reproductive numbers allow extremely tight packing of species on the $R$ axis. This pathology vanishes in more realistic models with finite populations that run the risk of demographic extinction (Buttel, 2001), as well as in models where the strict hierar- chy is relaxed (Adler and Mosquera, 2000; Durrett and Levin, 1998).

Kinzig et al. (1999) showed that if introductions are made more rapidly, so that the assemblage does not have time to come to equilibrium between introductions, there is no limit to the amount of diversity that can be achieved, even within a fixed range of fecundities. In particular, they found that if a large number of species are introduced into an empty habitat at the same time, roughly half of them will survive to form an equilibrium assemblage (Dushoff et al. (submitted) provides theoretical support for this simulation result). This is a rather striking contrast with the logarithmic accumulation found when species are added one at a time. Again, of course, finite populations and demographic extinction prevent species diversity from actually increasing without bound (Buttel et al., in press).

One of the more remarkable aspects of these simple community assembly models is that, even as diversity increases, characteristic scaling laws emerge in the distribution of species abundances. In the simplest model (hierarchical competition with constant mortality) for example, the relationship between fecundity and cumulative occupancy can be approximated by a simple scaling relationship (Fig. $1)$. The exact form of this relationship changes for more realistic models, but still robust scaling laws emerge and are stabilitzed, as statistical outcomes of local competition.

Although this is a highly simplified model, it is interesting to explore how competition acts to struc-

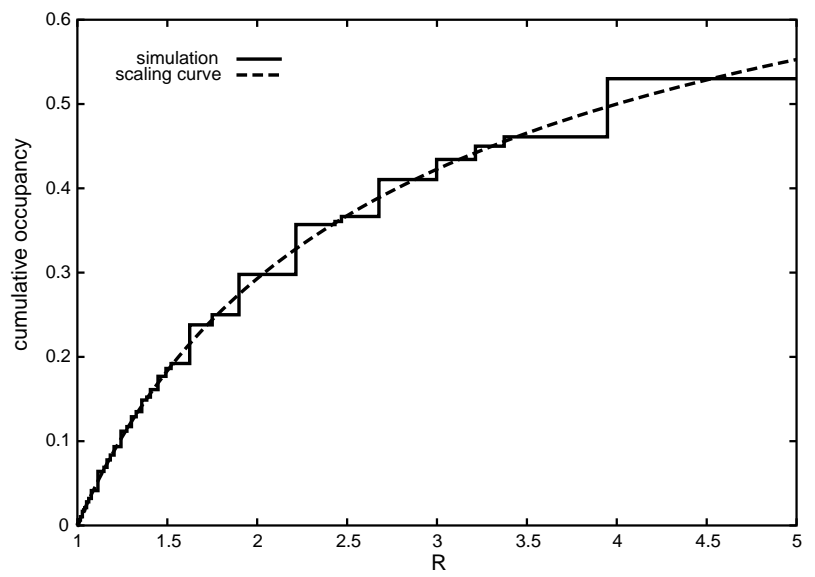

FIG. 1. - Cumulative occupancy below a given fecundity in a hierarchical model (with constant mortality), plotted together with the scaling relation $1-[1 /(R)]^{1 / 2}$, where $R=f / m$. This community was assembled using 1000 species chosen with $R=10^{U}$, where $U$ is a uniform random variate, and added sequentially (with inviable species allowed to go extinct after each introduction). The observed pattern (the vertical spike for each species intersecting the scaling curve) is the same for any assemblage of species. 
ture assemblages, and thereby to gain insight into real systems where the hierarchical model makes some sense. May and Nowak (1994) showed that a simple geometric model can predict which species will survive from a group of candidate species in the constant-fecundity model. Dushoff et al. (submitted) showed that, with a suitable transformation of parameters, the question of species assemblages in the constant-fecundity case is equivalent to that in the constant-mortality case, and in fact to a whole family of constraint curves. In general, survival of species $i$ will depend on two quantities: the amount of space occupied by its $i-1$ superior competitors

$$
\sigma_{i-1}=\sum_{j=1}^{i-1} p_{j}
$$

and the amount of displacement caused by the superior competitors

$$
\phi_{i-1}=\sum_{j=1}^{i-1} f_{i} p_{j} .
$$

For a family of curves including both constant mortality and constant fecundity as limiting cases, $\sigma$ and $\phi$ fall on a single curve for any equilibrium assemblage. That is, assemblages structure themselves in such a way that their net effect on 'downstream' competitors inferior to the bottom competitor can be predicted directly from the amount of space occupied, regardless of the actual composition of the species.

The geometric picture in this case is as follows. We consider species reproductive numbers on a transformed scale $z$ (e.g. in the constant mortality case, $z=\log R$ ). In any community, the top competitor is able to exclude inferior competitors for a distance along the $z$ axis exactly to the distance between its position and zero. That is, if the top competitor's rescaled reproductive number is $z_{1}$, no species will be able to survive in the 'competitive shadow' between $z_{1}$ and $2 z_{1}$. This pattern is repeated iteratively: each successive competitor casts a competitive shadow exactly equal to the amount of free space on the axis between it and the competitor to its left.

This geometric picture can be used to predict the outcome of serial or simultaneous introductions. It provides simple relations between occupancy, displacement and the end of competitive shadows, and sheds light on the structure of communities that develop with either simultaneous or successive introductions. Dushoff et al. (submitted) point out that the geometric picture is scale invariant with respect to behavior on the $z$ axis: the geometry tells us that the behavior of any portion of the axis that contains the origin can be scaled to the unit interval, and should behave exactly the same under the same pattern of introductions.

When many species are introduced uniformly on the $z$ axis, roughly half survive, and they are distributed approximately uniformly. When species are introduced one by one, however, a different sort of scale-invariant pattern emerges. It can be shown that the long-term probability of finding a species near point $z$ is proportional to $1 / z$. It follows that the number of species present grows only logarithmically with the number of species introduced.

The striking difference between the species densities obtained through sequential vs. simultaneous introduction points up the potential importance of the balance between speciation and extinction. In the sequential introduction case, May and Nowak (1994) and subsequent workers allowed the system to reach equilibrium between successive introductions. The results of the simultaneous introduction experiment show that, if introduction rates are high compared to the dynamics of population growth, some species that would go extinct may be 'rescued' by the introduction of other species which change the equilibrium structure and allow the threatened species to survive. Inferior competitors are in effect rescued from intermediate competitors by superior competitors. Intermediate competitors, with their higher reproductive numbers, exclude inferior competitors more effectively than superior ones can. If a superior competitor invades and takes space from an intermediate competitor, this may benefit inferior competitors. Of course, these three roles are relative, and a species may play all three within a single community.

In no case does this system develop a stable state that resists invasion. In fact, we know that on the $z$ axis the invasible range in front of each species, on the character axis, will be exactly as large as the uninvasible range in its competitive shadow. Thus we expect roughly half of all random introductions to successfully invade the system. Interestingly, though, the serial introduction system, uniform on $\log z$, is far more resistant to multiple, catastrophic extinction than the simultaneous introduction system, which is uniform on $z$. The increase in spacing between species with higher reproductive numbers limits the effect of new species on distant competitors, and provides the assemblage with a 'structural resiliency' that resists large-scale changes, even though it fails to resist to the establishment of new invaders. 
Simple models of this type can provide insights into the distribution of abundances within communities. They also tell us something about the evolution of resiliency, at least in terms of community composition. Clearly, however, more work is needed in understanding when and how resiliency evolves at a community level. Should we even expect communities to become more resilient through evolution, given that the relevant levels of selection are well below the community scale? This is an open question.

\section{SPECIES PERSISTENCE IN DYNAMIC LANDSCAPES}

The models of the previous section hypothesize a fixed landscape of patches, subject only to extinction and recolonization. Real landscapes, however, are dynamic, and patches themselves come and go. In a recent publication, Keymer et al. (2000) extended the metapopulation model to landscapes where habitat is created and destroyed dynamically. In this section we propose that the problem of community invasibility and resilience described in the preceding section is equivalent (in the mean field) to the problem of metapopulation persistence in dynamic landscapes (sensu Keymer et al., 2000). Therefore invasion success can be determined by representing the community resident community (prior to invasion) as a dynamic landscape of habitable sites (patch dynamics). Invasion will occur only if the invader is able to persist in the dynamic landscape representing the resident community.

\section{Metapopulation persistence in dynamic landscapes}

Although Keymer et al. (2000) start with a fully spatial stochastic process, in the mean-field approximation metapopulation dynamics can be described by an occupancy function that represents the proportion of occupied sites and follows Levins' (1969) laws of metapopulation dynamics. The population dynamics, however, depend on the patch dynamics - changes in the proportion of habitable 'patches' (or sites available for occupancy). Nonhabitable sites are restored at rate $\lambda$, while habitable sites are destroyed or degraded at rate $e$ (thus $1 / e$ gives the mean lifespan of a habitable patch). If $p$ is the proportion of sites that are occupied, and $h$ is the proportion that contain suitable habitat, the dynamics are given by:

$$
\begin{gathered}
\frac{d p}{d t}=f p(h-p)-(m+e) p \\
\frac{d h}{d t}=\lambda(1-h)-e h .
\end{gathered}
$$

The patch dynamics (Eq. 6) are independent of the population dynamics (Eq. 5), and thus will converge independently. If we allow patch dynamics to reach equilibrium, we can write the basic reproductive number for an invading species in terms of two properties of the patch-dynamics regime: the equilibrium amount of habitat $\hat{h}=\lambda(\lambda+\mathrm{e})$, and the equilibrium patch-destruction rate $\hat{e}$ :

$$
\tilde{R}=\frac{f \tilde{h}}{m+e} .
$$

The population will persist when $\tilde{R}>1$. Thus, the amount of successful colonization $(f \hat{h})$ from occupied patches must be greater than the rate of clearance $(m+e)$. The condition $\tilde{R}>1$ determines the possible dynamic landscapes (in terms of $e$ and $\hat{h}$ ) in which a given species is able to persist. When the species does persist, the equilibrium occupancy is given by a modified version of the standard metapopulation equation:

$$
\hat{p}=\hat{h}(1-1 / \tilde{R})
$$

\section{The resident community as a dynamical landscape}

Consider a hierarchical community of the form described in the section 3 (Eq. 1). From the point of view of an invader species $i$, we can define every site occupied by better competitors as a destroyed site. Thus, the amount of available habitat (for the invader) is

$$
\hat{h}_{i}=1-\sigma_{i-1} .
$$

Since the resident species are at a dynamic equilibrium, available patches are created by clearance of superior competitors (disturbance) and destroyed by colonization of superior competitors. Thus, the destruction rate of habitat patches from the invader's point of view is

$$
e_{i}=\phi_{i-1}
$$

Hence, the dynamics of the hierarchical community are equivalent to those of a metapopulation under a patch-dynamic regime. The regime of patch dynamics is a property of the resident community (Eqs. 9, 10) and invasibility is a property resulting from the interaction of residents and invader. 


\section{Community invasibility as species persistence in dynamic landscapes}

Equations 9 and 10 allow us to see a hierarchical community (Eq. 1) as a dynamic landscape (Eq. 6) characterized by patch availability and mean lifespan (1/e). In this framework, the community invasibility problem is equivalent to the problem of metapopulation persistence in a dynamic landscape, and the dynamics of invasion is equivalent to metapopulation dynamics (Eqs. 7, 8). Therefore, we know that a resident community will be successfully invaded by species $i$ if and only if

$$
\tilde{R}_{i}=\frac{f_{i} \tilde{h}_{i}}{m_{i}+e_{i}}>1
$$

\section{The two species case}

Consider the situation of two species sequentially invading a pristine environment. The first invader finds an empty community $\left(\hat{e}_{1}, \hat{h}_{1}\right)$ represented by a static landscape $(\hat{e}=0)$ with all sites being habitat patches $(\hat{h}=1)$. Therefore, the condition for this founder species to invade is equivalent to the metapopulation persistence in static landscapes

$$
f_{1}>m_{1}
$$

The second species does not neccesarily find an "empty community". If species 2 is a poorer competitor than species 1 then the environment $\left(\hat{e}_{2}, \hat{h}_{2}\right)$ is no longer pristine. Species 2 instead encounters a much "destroyed" (sensu Bascompte and Sole, 1998) and "ephemeral" (sensu Keymer et al., 2000) habitat and therefore will invade it only if

$$
f_{2} \hat{h}_{2}>\mathrm{m}_{2}+\hat{e}_{2} .
$$

In other words, invasion will occur if the effective colonization rate is greater than the combined rate of clearance due to intrinsic mortality and patch destruction (invasion by superior competitors).

Following Kinzig et al. (1999) we can also think about this problem as a species-packing problem in niche space. If we write the fundamental rate of increase $r_{i}=f_{i}-m_{i}$, we can represent niche separation between the two species (in $R$ space) as the ratio between their reproductive numbers:

$$
\frac{R_{2}}{R_{1}}>1+\frac{r_{1}}{m_{2}} .
$$

Thus, there is a necessary separation between reproductive numbers before an invader can be successful. This is because of the reduction in available habitat, and the losses due to invasion from superior competitors. If we fix the parameters of the resident $\left(R_{1}\right.$ and $\left.r_{1}\right)$, we see that the invader can increase success either by increasing its reproductive number $R_{2}$, or by decreasing its life span $1 / m_{2}$. This is because, for fixed $R$, shorter-lived species will have faster reproduction, and thus be more effective colonizers.

\section{Relaxing the hierarchy}

Although we focus our analysis here on hierarchical competitive structures, more general cases with non-transitive relationships (Buss and Jackson, 1979) can be incorporated into the patch-dynamic framework by representing communities as landscapes with habitat-quality distributions where patches occupied by stronger competitors are represented as poor-quality habitat, and conversely. In this paper we presented the simplest case of landscape dynamics as patch creation and destruction and showed its equivalence with a hierarchically structured community. More general models of competition for space (sensu Durrett and Levin, 1998) can be represented in the mean field as more complicated cases of patch dynamics. A very promising challenge is to extend this formalism to community dynamics and evolution in spatially explicit frameworks, with more general dispersal (Travis and French, 2000) and competition (Adler and Mosquera, 2000) functions. In these scenarios (spatially dependent) patch quality will represent the resident community's state.

It is clear that more theoretical studies on spatially dependent patch dynamics and metapopulation persistence in dynamic landscapes, and on scale invariance and the emergence of ecosystem patterns, is urgently needed for a better understanding of spatially distributed multispecies communities. However, further study of the implications of scale invariance in simple hierarchical models to the emergence of ecosystem patterns is also worthwhile.

\section{FOOD WEBS, TROPHIC CASCADES AND RESOURSE TRANSFORMATION NETWORKS}

In 1968, Ramón Margalef (p. 81) wrote: “The energy gates at the places where species interact - or where they interact with environment - are the 
organs by which selection is achieved and evolution occurs, the rate of evolution depending on the efficiency of the gate".

Margalef taught us to think about evolution within the framework of ecosystem organization, thus we must extend previous models of space utilization (sections 3 and 4) to incorporate assumptions about nutrient (or biomass) pathways (nutrient utilization). In this new scenario, different spatial patterns of nutrient utilization (at the ecosystem level) are seen as the result of diffusive coevolution operating at the level of individual organisms. Although is clear that more realistic models of spatial competition are needed, it is also worthwhile to extend simple spatial models to incorporate nutrient utilization.

\section{The role of community structure in shaping ecosystem patterns}

Patch occupancy is a natural way to build a theoretical bridge linking the landscape and ecosystem criteria (sensu Allen and Hoekstra, 1992). The question of the units of selection and spatial localization of interactions (Durrett and Levin, 1994) would determine critical scales at which patch occupancy would be meaningful for ecosystem function. In this way, we can think of a patch large enough to hold one organism as the locus for biogeochemical transformations. Thus, organisms, their populations and communities (sometimes nested other organisms) play a catalytic role for such transformations. Thus, scale-invariant patterns of spatial occupancy could be translated into spatio-temporal patterns of nutrient distribution - ecosystem pattern. Recent landscape ecology studies such as the spatiotemporal analysis of nitrogen dynamics in forest watersheds (Bartell and Brenkert, 1990) as well as spatiotemporally embedded food webs (Holt, 1996a,b; Polis et $a l ., 1996)$ point in directions envisioned by Margalef in the late 1960s.

\section{THE DYNAMICS OF ECOSYSTEMS: REDFIELD RATIOS AND NUTRIENT CYCLES}

The earlier sections of this paper have examined the evolution of populations in relation to one another, and demonstrated that characteristic patterns can arise in the distribution of species. From simplistic assumptions that reflect nothing more than competition for space, and the evolutionary dynamics that this implies, robust patterns can emerge from the self-organization of ecological communities (Chave et al., 2001). This is only part of the story, however. The dynamics of competition among species, and indeed the dynamics of persistence of individual populations, are mediated by the physical and chemical environment - by the availability of nutrients (and of water in intertidal and terrestrial systems), as well as by factors such as temperature and salinity. In turn, the biotic composition of ecosystems fundamentally affects the ambient physico-chemical context in which populations evolve. Thus, populations together with their physical and chemical environments constitute interconnected feedback systems, namely ecosystems.

It does not detract from the notion of the ecosystem to observe that ecosystems are open systems, whose definition hence is subject to a great deal of arbitrariness. Ecosystems are not evolutionary units, selected for their macroscopic features because those features benefit the constituent populations. Rather, what patterns there are must emerge from the self-organization of components whose evolutionary context is far broader than that of a single ecosystem. The statistical mechanics of the assembly of communities must rest heavily on various laws of large numbers, producing pictures that are to large extent insensitive to many of the fine details, providing therefore an environment whose features are sufficiently predictable to facilitate adaptation and survival.

Community ecology concerns itself largely with patterns in the distribution of species; ecosystem ecology concerns itself further with the fluxes of materials such as key elements, and with patterns in their distribution. Integrating these two aspects, the biotic and the abiotic, is the essence of understanding ecosystems, and involves the interplay of processes across diverse scales of space, time and organizational complexity.

Life depends on a diversity of elements, but carbon $(\mathrm{C})$, nitrogen $(\mathrm{N})$ and phosphorus $(\mathrm{P})$ are at the core, along with the constituents of water itself, hydrogen $(\mathrm{H})$ and oxygen $(\mathrm{O})$. In 1934, the young animal physiologist Albert Redfield observed that $\mathrm{C}$, $\mathrm{N}$ and $\mathrm{P}$ in marine plankton occurred in remarkably constant proportions, 106:16:1, across a diverse range of environments (Redfield, 1934). He further noted that the $\mathrm{N}: \mathrm{P}$ ratio was very similar to those found in dissolved inorganic form in seawater, and proposed that the plankton actually control the nitrate:phosphate levels in the ocean through remineralization (Falkowski, 2001). 
The situation is not as simple as envisaged by Redfield. Indeed, later work showed that the Redfield ratios are indeed approximately constant across a wide range of environments, and that plankton ratios match ambient levels very closely, despite great variation in the absolute concentrations. Redfield espoused the view that phosphate limits ocean productivity, but modern work (Falkowski, 1997; Karl et al., 1997) argues that nitrogen can be limiting, because nitrogen fixation has high iron demands. The activities of marine plankton surely influence the cycles of $\mathrm{C}, \mathrm{N}$ and $\mathrm{P}$, but in turn are controlled by the availability of those nutrients and others in the marine environment. Furthermore, although there is a great deal of similarity in the element ratios of plankton, there are differences. That is, different species utilize resources in different ways, a partial answer to the conundrum of how so many species of plankton manage to coexist (Hutchinson, 1961). Thus to understand the constancy of the element ratios, one cannot simply take the viewpoint that the ambient ratios represent what is remineralized from the plankton. The plankton and the water column are involved in an ongoing colloquy in which each is influenced by the other, within a turbulent environment characterized by incomplete mixing, and the infusion of nutrients from exogenous sources.

Why then do constant element ratios emerge, given the considerable heterogeneity in environmental conditions, as well as biological diversity? Such questions direct attention to how such ratios can emerge, and be stabilized, in the face of long-term evolutionary change. How too can so much biotic diversity be sustained, given such constancy in key element ratios? The usual focus is on marine systems, but it is also instructive to ask whether such patterns can be seen in freshwater, or in terrestrial systems. For large lakes, one does indeed see similar ratios, but smaller lakes deviate from the canonical values (Downing and McCauley, 1992). Forests present even greater challenges, even in terms of determining what measurements would be comparable.

Other factors also complicate analysis. Lars Hedin, Pamela Matson and Peter Vitousek have been comparing the biogeochemistry of Hawaiian forests across a spectrum ranging from very young forests (300 years old) to very old ones (more than 4 million years). They show that these forests in their early stages of development receive much $\mathrm{P}$ from weathering, and are primarily nitrogen limited, but as forests age, $\mathrm{P}$ becomes progressively the limiting nutrient (Hedin et al., submitted). Thus external factors play a crucial role, especially for younger forests, in determining $\mathrm{N}: \mathrm{P}$ ratios. Marine and terrestrial systems differ fundamentally in terms of how closed they are, how well mixed they are, and how mature they are evolutionarily. These factors are interrelated of course; the limited mixing in terrestrial environments, say among the various Hawaiian Islands, permits local exogenous heterogeneity to exert a greater influence. The challenge is to tease apart these influences, and to determine how much of the pattern we see is exogenously driven, and how much is the result of self-organization. Furthermore, how different should the ratios of elements be in a system in its early stages of development from one that has seen millions of years of evolution?

To address issues of this sort, one must start from the bottom up, not from the top down. The individual is where the gene meets the environment, and hence the individual is the primary unit of selection. Valid arguments can be made, of course, to treat the gene itself as the unit of selection (Dawkins, 1982); in other cases, such as for the social insects, the operational unit may involve collections of individuals within a species. Tight interspecific linkages, such as in host-parasite systems, can introduce even higher-level considerations; but these are all a far cry from the ecosystem. With few exceptions, tight coevolution among species is hard to find in Nature (Futuyma and Slatkin, 1983), and we must understand what patterns we do detect at the macroscopic level as having emerged from the collective and selfish evolution of its individual species. These emergent patterns still feed back to influence evolution at lower levels, but the coevolutionary influences are what Ehrlich and Raven (1964) termed "diffuse".

Our agenda, then, in future work, will be to extend the approach of the previous sections to examine the evolution of patterns of resource uptake, nitrogen fixation, chelation and other mechanisms for resource use and sequestration, in order to elucidate how patterns of element cycling emerge. As in the previous sections, an adaptive dynamics is needed that addresses the competitive and other interactions among genetically diverse types, sharing a global commons in which each individual's actions has a perhaps small effect globally, but where the collective actions of many individuals assumes great importance. This is not much different than the problems we face in understanding the threats to human sustainability from the collective actions of billions of individuals, each of whom sees 
his or her actions as having minimal environmental impact (Levin, 1999). Thus the relevant models should be individual-based, or at least represent the statistical mechanics of ensembles of such individuals. They should be spatially explicit, because the localization of interactions is an essential and overriding evolutionary truth. And ultimately, from these overly detailed models, it will be essential to derive robust macroscopic features that do not depend upon the intricate details. It is those regularities that impel such investigations, and that must also be the endpoints.

\section{ACKNOWLEDGMENTS}

We are pleased to acknowledge the support of the National Science Foundation, award DEB-0083566, The David and Lucile Packard Foundation, awards 8910-48190, 7426-2208-1, and the Andrew W. Mellon Foundation.

\section{REFERENCES}

Adler, F.R. and J. Mosquera. - 2000. Is space necessary? Interference competition and limits to biodiversity. Ecology, 81: 3226-3232.

Allen, T.F.H. and T.W. Hoekstra. - 1992. Toward a unified ecology. University Press, New York.

Armstrong, R.A. and R. McGehee. - 1980. Competitive exclusion. Am. Nat., 115: 151-170.

Bartell, S.B. and A.L. Brenkert. - 1990. A spatial-temporal model of nitrogen dynamics in a deciduous forest watershed In: M.G Turner and R.H. Gardner (eds.), Quantitative methods in landscape ecology, pp. 379-398. Springer-Verlag, New York.

Bascompte, J. and R. Sole. - 1998. Models of habitat fragmentation. In: Modeling spatio-temporal dynamics in ecology. SpringerVerlag, Berlin.

Bolker, B.M., and S.W. Pacala. - 1999. Spatial moment equations for plant competition: Understanding spatial strategies and the advantages of short dispersal. Am. Nat., 153: 575-602.

Buss, L.W. and J.B.C. Jackson. - 1979. Competitive networks: nontransitive competitive relationships in criptive coral reef environments. Am. Nat., 113: 223-234

Buttel, L., R. Durrett and S.A. Levin. - (in press). Competition and Species Packing in Patchy Environments. Theor. Pop. Bio.

Chave, J., H.C. Muller-Landau and S.A. Levin. - (submitted). Comparing classical community models: Theoretical consequences for patterns of diversity. Am. Nat.

Dawkins, R. - 1982. The Extended Phenotype: The Gene as the Unit of Seclection. W.H. Freeman, Oxford.

Downing, J.A. and E. McCauley. - 1992. The nitrogen: phosphorus relationship in lakes. Limnol. Oceanogr., 37: 936-945.

Durrett, R., and S.A. Levin. - 1994. The importance of being discrete (and spatial). Theor. Pop. Biol., 46: 363-394.

Durrett, R., and S.A. Levin. - 1998. Spatial aspects of interspecific competition. Theor. Pop. Biol., 52: 30-43.

Dushoff, J., L. Worden, S.A. Levin and J.E. Keymer. - (submitted) Scale invariance in community assembly.

Ehrlich, P.R. and P.H. Raven. - 1964. Butterflies and plants - a study in coevolution. Evolution, 18: 586-608.

Falkowski, P. - 1997. Evolution of the nitrogen cycle and its influence on the biological sequestration of $\mathrm{CO}_{2}$ in the ocean Nature, 387: 272-275.

Falkowski, P.G. - 2001. Rationalizing elemental rations in unicellular algae. J. Physiol., 36: 3-6.
Futuyma, D. and M. Slatkin (eds.). - 1983. Coevolution. Sinauer, Sunderland, MA

Hastings, A. - 1980. Disturbance, Coexistence, History, and Competition for Space. Theor. Pop. Biol., 18: 363-373.

Hedin, L.O., P.M. Vitousek and P.A. Matson. - (submitted). Pathways and implications of nutrient loss during four million years of tropical forest ecosystem development. Ecology.

Holt, R.D. - 1996a. Food webs in space: an island biogeographic perspective. In: G. Polis and K. Winemiller (eds), Food webs: integration of patterns and dynamics. Chapman and Hall, New York.

Holt, R.D. - 1996b. Temporal and spatial aspects of food web structure and dynamics. In: G. Polis and K. Winemiller (eds), Food webs: integration of patterns and dynamics. Chapman and Hall, New York.

Horn, H.S. and R.S. MacArthur. - 1972. Competition among fugitive species in a harlequin environment. Ecology, 53: 749-752.

Hubbell, S.P. - 1997. A unified theory of biogeography and relative species abundance and its application to tropical rain forests and coral reefs. Coral Reefs, 16: S9-S21.

Hubbell, S.P. - 2001. A Unified Neutral Theory of Biogeography. Princeton University Press.

Hutchinson, G.E. 1961. The paradox of the plankton. Am. Nat., 95: 137-145.

Karl, D., R. Letelier, L. Tupas, J. Dore, J. Christian and D. Hebel. 1997. The role of nitrogen fixation in biogeochemical cycling in the subtropical North Pacific Ocean. Nature, 388: 533-538.

Keymer, J.E., P.A. Marquet, J.X. Velasco-Hernandez and S.A. Levin. - 2000. Extinction thresholds and metapopulation persistance in dynamic landscapes. Am. Nat., 156: 478-494.

Kinzig, A.P., S.A. Levin, J. Dushoff and S. Pacala. - 1999. Limiting similarity and species packing in competition-colonization mModels. Am. Nat., 153: 371-383.

Levin, S.A. - 1970. Community equilibria and stability, and an extension of the competitive exclusion principle. Am. Nat., 104: 413-23.

Levin, S.A., and R.T. Paine. - 1974. Disturbance, patch formation, and community structure. Proc. Natl. Acad. Sci., 71: 2744-2747.

Levin, S.A. - 1998. Ecosystems and the biosphere as complex adaptive systems. Ecosystems, 1: 431-436.

Levin, S.A. - 1999. Fragile Dominion: Complexity and the Commons. Perseus Books Group, Reading, MA.

Levins, R. - 1968. Evolution in changing environments. Princeton U. Press, Princeton.

Levins, R. - 1969. Some demographic and genetic consequences of environmental heterogeneity for biological control. Bull. Entomol. Ass. Am., 15: 237-240.

Levins, R., and D. Culver. - 1971. Regional coexistence of species and competition between rare species. Proc. Nat. Acad. Sci., 68: 1246-1248.

MacArthur, R.H., and R. Levins. - 1967. The limiting similarity, convergence and divergence of coexisting species. Am. Nat., 101: 377-385.

Margalef, R. - 1968. Perspectives in ecological theory. The University of Chicago Press, Chicago.

May, R.M. and M.A. Nowak. - 1994. Superinfection, metapopulation dynamics and the evolution of diversity. J. Theor. Biol., 170: 95-114.

Nowak, M. and R. May. - 1994. Superinfection and the evolution of parasite virulence. Proc. R. Soc. B, 55: 81-89.

Paine, R.T. and S.A. Levin. - 1981. Intertidal landscapes: disturbance and the dynamics of pattern. Ecol. Monogr., 51: 145-178.

Polis, G.A., R.D. Holt, B.A. Menge and K. Winemiller. - 1996. Time, space, and life historiy: influences on food webs. In: G. Polis and K. Winemiller (eds.), Food webs: integration of patterns and dynamics. Chapman and Hall, New York.

Quinn, J.F. - 1982. Competitive hierarchies in marine benthic communitites. Oecologia, 54: 129-135.

Redfield, A.C. -1934 . On the proportions of organic derivatives in sea water and their relation to the composition of plankton. In: R.J. Daniel (ed.), James Johnstone Memorial Volume, pp. 177192. University Press of Liverpool.

Tilman, D. -1990 . Constraints and tradeoffs: towards a predictive theory of competition and succession. Oikos, 58: 3-15.

Tilman, D. - 1994. Competition and biodiversity in spatially structured habitats. Ecology, 75: 2-16.

Travis, J.M.J. and D.R. French. - 2000. Dispersal functions and spatial models: expanding our dispersal toolbox. Ecol. Lett., 3: 163-165. 